\title{
Hybrid Speckle Noise Reduction Method for Abdominal Circumference Segmentation of Fetal Ultrasound Images
}

\author{
Fajar Astuti Hermawati ${ }^{1}$, Handayani Tjandrasa ${ }^{2}$, Nanik Suciati ${ }^{3}$ \\ ${ }^{1,2,3}$ Department of Informatics, Institut Teknologi Sepuluh Nopember, Surabaya, Indonesia \\ ${ }^{1}$ Department of Informatics, Universitas 17 Agustus 1945, Surabaya, Indonesia
}

\section{Article Info \\ Article history: \\ Received Sep 17, 2017 \\ Revised Mar 12, 2018 \\ Accepted Mar 20, 2018}

\section{Keyword:}

Abdominal circumference Automatic segmentation Image denoising Specklenoise Ultrasound image

\begin{abstract}
Fetal biometric size such as abdominal circumference (AC) is used to predict fetal weight or gestational age in ultrasound images. The automatic biometric measurement can improve efficiency in the ultrasonography examination workflow. The unclear boundaries of the abdomen image and the speckle noise presence are the challenges for the automated AC measurement techniques. The main problem to improve the accuracy of the automatic AC segmentation is how to remove noise while retaining the boundary features of objects. In this paper, we proposed a hybrid ultrasound image denoising framework which was a combination of spatial-based filtering method and multiresolution based method. In this technique, an ultrasound image was decomposed into subbands using wavelet transform. A thresholding technique and the anisotropic diffusion method were applied to the detail subbands, at the same time the bilateral filtering modified the approximation subband. The proposed denoising approach had the best performance in the edge preservation level and could improve the accuracy of the abdominal circumference segmentation.
\end{abstract}

Copyright () 2018 Institute of Advanced Engineering and Science. All rights reserved.

\section{Corresponding Author:}

Fajar Astuti Hermawati,

Department of Informatics,

Institut Teknologi Sepuluh Nopember Surabaya,

Jl. Teknik Kimia, Gedung Teknik Informatika, Kampus ITS Sukolilo, Surabaya, 60111, Indonesia.

Email: fajar.astuti15@mhs.if.its.ac.id

\section{INTRODUCTION}

The medical ultrasound is a diagnostic tool to provide images of organs and structures non invasively. Compared to other modalities such as CT scans and MRI, the ultrasound has the lower price, easy to move (portable), small and easily manipulated, and non-ionizing [1], [2]. Besides being used to monitor pregnancy, the ultrasonography is widely used for examination of diseases such as liver cancer [3], cardiovascular disease [4], breast cancer [5], [6], and musculoskeletal disorders [7]. Nevertheless, there are many drawbacks of this modality such as the artefacts on ultrasound images that named speckle noise. The presence of the noise will lead to the difficulty of further analysis of ultrasound images.

According to [8], a segmentation technique to obtain the fetal biometric measurement automatically on an ultrasound image could improve the efficiency of the clinical workflow. The abdominal circumference (AC) is fetal biometric size that used to confirm the gestational age, to estimate the fetal weight, and to identify the growth patterns and the abnormalities of the fetus. The existing studies of the AC segmentation technique included the edge detector approach, clustering technique, Hough transform, and gradient vector field snake (GVF) method [9]-[11]. In this work, the active geometric shape model (AGSM) approach that proposed by [1] was applied to segment the AC automatically. The AGSM could identify various geometric shapes of lines, circles, ellipse and cubic splines. The advantage of the AGSM method was robust to noise, broken lines and the non-uniform contrast [12]. The fetal abdomen image has no clear boundaries and has 
inconsistencies in the internal structure [13]. Moreover, an ultrasound image often contains speckle noise. So it takes a preprocessing technique called the speckle noise reduction method that aims to elimin ate noise while maintaining the feature of the boundary.

The speckle appears as a grain pattern that varies depending on the type of biological tissue. According to Joel \& Sivakumar [14], there were two groups of the speckle noise reduction methods, i.e. the spatial filtering method and the multiscale or multiresolution method. Loizou \& Pattichis [15] divided the spatial filtering techniques into three groups, i.e. the linear filtering approach such as the Lee filter [16] and the Frost filter [17], the nonlinear filtering technique such as the bilateral filter [18] and the shock filter [3], and the diffusion filtering method. The diffusion filtering techniques, such as the conventional anisotropic diffusion method [19] and the speckle reduction anisotropic diffusion (SRAD) method [20], adopted the partial differential equations and set up an adaptive filtering constraint in the denoising process. The SRAD approachwas the development of the anisotropic diffusion method. The last group of the speckle reduction methods was the multiresolution based method that one of which implements a wavelet transform. The wavelet based technique proposed by Babu et al [21] was used to remove the noise speckle from the SAR image. Whereas, Gopinathan et al [22] suggested the wavelet based denoising scheme to eliminate Gaussian noise. Hamiane \& Saeed [23] applied the discrete wavelet transform to reduce noise in MRI brain images.

From all of the denoising methods, the SRAD is the most recent and widely developed technique. The SRAD is not only able to maintain the edge of the image but also able to increase the detail image. However, the SRAD is not able to eliminate the noise in a high-intensity area. Some hybrid techniques were proposed to overcome the denoising problem in the high-intensity area of an image. One of the hybrid denoising techniques was the multiresolution bilateral filter which combining the wavelet thresholding approach and bilateral filtering method [24], [25]. Zhang et al [25] applied the fast bilateral filtering to decrease the computational time of the technique proposed by Zhang \& Gunturk [24].

In this paper, we propose a hybrid denoising framework that combining the multiresolution bilateral filtering method and the anisotropic diffusion method. To reduce speckle noise in the high-intensity area, we apply wavelet decomposition method that divides the image signal into different resolutions, i.e. an approximation subband and three detail subbands which represent the high-intensity area. We implement the anisotropic diffusion method in the detail resolution images to reduce noise better. This work does not only propose a speckle noise reduction method but also compare it to the other speckle noise reduction methods. This research also shows the influence of the proposed noise reduction technique in increasing the accuracy of the AC segmentation in the fetal ultrasound images.

The paper is arranged into four sections. In Section 1, we explain the problem that will be resolved and the literature review about the speckle noise reduction methods. Section 2 describes the research method that includes the existing denoising methods, the proposed denoising scheme, the image dataset, the AC segmentation method and the performance measurement. In Section 3, we analyze the experiment result, i.e. the noise reduction test and the segmentation test. Finally, Section 4 includes the conclusion of this work.

\section{RESEARCH METHOD}

We conducted two experiments to evaluate the proposed speckle noise reduction method as follows: (1) noise reduction experiment and (2) abdominal circumference segmentation experiment. The objective of the first test is to evaluate the capability of the proposed method in reducing the speckle noise and maintaining the edge. The second trial aims to show the influence of noise reduction techniques in the accuracy improvement of the abdominal circumference segmentation on the ultrasound image. In this section, we present a research method that it includes the existing despeckling techniques, the data, the proposed method, the fetal abdomen segmentation, and the performance evaluation technique.

\subsection{Existing denoising methods} as follows :

Firstly, we introduce the existing despeckling methods that are compared to the proposed approach

a. Lee filter [16]

Lee filter aimsto eliminatethe noise in a radar image while maintaining edges or features. This filter isbased on the linear filter model and the least mean square error approximation. Since, $I_{x, y}$ is the pixelintensityat the coordinates $(x, y)$ in a $M \times N$ noisy image and $\aleph_{x, y} \in I_{x, y}$ are pixels in a $n \times n$ neighborhood window, and $m_{s}$ is the mean of the intensities in the window $\aleph$, the Lee filter is expressed in the equation as follows :

$$
\widehat{\aleph}_{x, y}=m_{s}+k_{s}\left(\aleph_{x, y}-m_{s}\right)
$$


The variable $k_{\mathrm{s}}$ in Equation (1) is an adaptive filter coefficient that defined in the equation as follows:

$$
k_{s}=1-\frac{c_{u}^{2}}{c_{s}^{2}}
$$

with $C_{s}^{2}=\left(\frac{1}{(n x n)}\right) \frac{\sum_{i, j=1}^{n}\left(s_{i, j}-m_{s}\right)^{2}}{\left(s_{i, j}-m_{s}\right)^{2}}$ and $C_{u}^{2}=\frac{\operatorname{var}\left(z^{\prime}\right)}{\left(\overline{z^{\prime}}\right)^{2}}$, wherevar $\left(z^{\prime}\right)$ and $\overline{z^{\prime}}$ are the variance and the mean of a homogeneous area in an image.

b. Frost filter [17]

The Frost filter usesa convolutional kernel that adapted to edge-containing regions by applying local statistics. The Frost filter is expressed by the following equation :

$$
\widehat{\aleph}_{p}=\sum_{q \in \mathrm{\aleph}_{x, y}} \frac{\mathrm{e}^{\left(-\mathrm{K} C_{p}^{2} d_{p, q}\right)}}{\sum_{q \in \mathrm{N}_{x, y}} e^{\left(-\mathrm{K} c_{p}^{2} d_{p, q)}\right.}} \cdot \aleph_{p}
$$

where $d_{p, q}$ is the distance from pixelpat coordinate $\left(i_{p}, j_{p}\right)$ to pixel $q$ at position $\left(i_{q}, j_{q}\right)$, with $p, q \in \aleph_{x, y}, \aleph_{x, y}$ is the $n \times n$ neighbor window, that is expressed in the following equation:

$$
d_{p, q}=\sqrt{\left(i_{p}-i_{q}\right)^{2}-\left(j_{p}-j_{q}\right)^{2}}
$$

The variable $\mathrm{K}$ is the damping factor chosen such that the homogenous area $K C_{s}{ }^{2}$ close to zero.

c. Bilateral filtering [18]

The bilateral filtering is a nonlinear filtering technique that can eliminate noise while preserving the edge of the image. Moreover, this filter implements the average of image intensities in a neighborhood window in a similar way to Gaussian filtering. To maintain the edge during the smoothing process, the bilateral method calculates the difference in a pixel intensity with its neighboring pixels. The following equation is used to express the bilateral function.

$$
\hat{I}(p)=\frac{1}{\mathrm{C}} \sum_{q \in \mathrm{N}(p)}\left(G_{\sigma_{d}}(\|q-p\|) \cdot G_{\sigma_{r}}(\|I(q)-I(p)\|)\right) \cdot I(q)
$$

where $I(p)$ is an input image and $\aleph(p)$ is a local window centered at the pixel $p$, while $q \in \aleph$ is the other pixel in the window $\aleph$. The function $G_{\sigma}($.$) is a Gaussian function with \sigma$ as its standard deviation. The value $\sigma_{d}$ and $\sigma_{\mathrm{r}}$ are parameters controlling the amount of the filtering in the image domains, and $C$ is a constant to normalize the function such that the sum of weights equal to 1.The control parameters for denoising applications are determined using a linear relationship between $\sigma_{r}$ and $\sigma_{n}$, where $\sigma_{n}$ is a noise standard deviation.

\section{d. Shock filter [3]}

Shock filter is based on a morphological operator, dilation or erosion, applied locally. The dilation or the erosion application dependson whether the pixel belongs. The influence zones, the maximum or minimum zone, aredetermined by the sign function. The sign function called the signum, $s$, in set $\{-1,0$, $+1\}$ is based on the Laplace operator. The border line between two influence zones produces a sharp discontinuity called shock.Shock filters applythe partial differential equation that run repeatedly as $n$ iterations. Leta noisy image $f(x, y)$ and $t=1,2, \ldots, n$. Thefiltered image equation at the $t$-th iterationis expressed as follows :

$$
u(x, y ; t)=-\operatorname{sign}(\Delta u(x, y ; t)|\nabla u(x, y ; t)|
$$

where $\nabla u(x, y ; t)=\left(u_{x}, u_{y}\right)^{t}$ is the spatial gradient of the function $u$ at vertical direction $\left(u_{x}\right)$ and at horizontal direction $\left(u_{y}\right)$, and $|\nabla u(x, y ; t)|=\sqrt{u_{x}^{2}+u_{y}^{2}}$. The function $\Delta u(x, y ; t)$ is modified using the coherenceenhancing shock filters that is proposed by [26], such that the Equation (6) becomes:

$$
\begin{aligned}
& u(x, y ; t)=-\operatorname{sign}\left(v_{w w}\right)|\nabla u(x, y ; t)| \\
& v_{w w}=c^{2} v_{x x}+2 c s v_{x y}+s^{2} v_{y y}
\end{aligned}
$$


where $v=K_{\sigma} * u$, and $w$ is the normalized dominant eigen vector of the structure tensor $J_{\rho}(\nabla u)=\left[\begin{array}{ll}v_{x x} & v_{x y} \\ v_{y x} & v_{y y}\end{array}\right]$, and $v_{x x}, v_{x y}, v_{y y}$ are the second order derivatives of $v$.

e. Anisotropic diffusion [19] and SRAD [20]

The SRAD method is an extension of the anisotropic diffusion approach. The anisotropic diffusion method uses the partial differential equation (PDE) approach which is a nonlinear filtering method that is expressed by the following equation:

$$
\frac{\partial f(i, j)}{\partial n}=\operatorname{div}(c(x, y, z, t) \nabla f(i, j)=c(x, y, z, t) \Delta f(i, j)+\nabla c \circ \nabla f(i, j)
$$

where $n$ is the number of iterations, $f(i, j)$ is an original image functionin the $(i, j)$ coordinates and $c(x, y, z, t)$ is the diffusion coefficient with the horizontal, vertical and diagonal directionat the $t$ th iteration. The diffusion coefficient allows to encourage the homogeneous image areasdiffusion and prevent the diffusion at the edges. The following equation expres ses the diffusion function that is used to enhance the higher contrast areas than, the lower ones.

$$
c(x, y, z, t)=e^{-(\nabla f(i, j) / k)^{2}}
$$

Conversely, the second diffusion function that affects thelarger areas is expressed as follows:

$$
c(x, y, z, t)=\frac{1}{\left(1+\left(\frac{\nabla f(i, j)}{\mathrm{k}}\right)^{2}\right)}
$$

where $k$ is a gradient threshold which controls the conduction. In the SRAD method, the $k$-value is estimated by the intensity average of a chosen homogenous area.At each step, the PDE is updated by the following equation :

$$
\nabla^{\prime} f(i, j)=\nabla f(i, j)+\Delta t . c(x, y, z, t)
$$

with $\Delta t$ is a integral constant, $0 \preccurlyeq \Delta t \preccurlyeq 1 / 7$.

\section{f. Decomposition wavelet [27]}

According to Sendur and Selesnick [27], there arethree main steps for the denoising algorithms using wavelet transforms, as shown in Figure 1. Firstly, the noisy image is analysed with discrete wavelet transforms (DWT). In this step, an input image is decomposed into the approximation subband using the lowpass filter (L) and the detail subbands using the highpass filter $(\mathrm{H})$. The detail subbands provide the detailed information in the vertical ( $\mathrm{LH})$, horizontal $(\mathrm{HL})$ and diagonal $(\mathrm{HH})$ directions of the original image. Furthermore, the wavelet coefficients in the detail subbands are modified using thresholding processes (T). The last step is the synthesis process using the inverse discrete wavelet transform (IDWT)

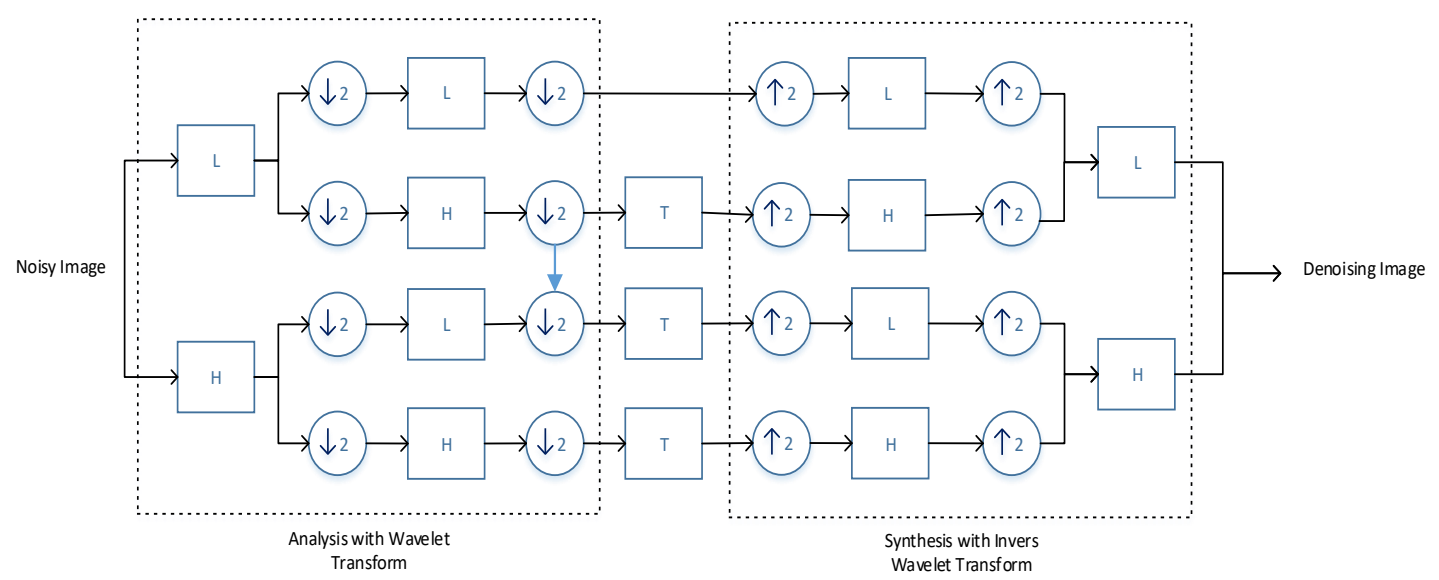

Figure 1. The block diagram of the wavelet thresholdingmethod 
The wavelet thresholding aims to eliminate the wavelet coefficients of the detail subbands that are lower than a particular threshold value. This process impacts on noise reduction without affecting the other essential features in the image. There are two thresholding methods including the soft thresholding and hard thresholding. In the soft thresholding procedure that is proposed by Donoho [28], the wavelet coefficients that smaller than the threshold value are set to zero, otherwise, the coefficients are subtracted from the threshold value. The following equation expresses the soft thresholding.

$$
w_{i}^{\prime}=\left\{\begin{array}{c}
0, \text { if }\left|w_{i}\right|<T \\
w_{i}-T, \quad \text { if } w_{i}>T \\
w_{i}+T, \quad \text { if } w_{i} \leq-T
\end{array}\right.
$$

The threshold value $T$ is estimated by the Bi variate shrinkage [27] using the following formula:

$$
T=\frac{\| \text { median }\left(w_{i}\right) \|}{0.6745}
$$

where $w_{i}$ is the diagonal wavelet coefficients of the $i$ th decomposition level.

g. Multiresolution bilateral filtering [24]

The multiresolution bilateral filteringis a hybrid method that integrates the multiresolution wavelet decomposition and bilateral filtering method. The bilateral filtering is appliedto the approximation subband of wavelet decomposition. Meanwhile, the detail sub-bands of wavelet decomposition are computed by the wavelet thresholding. The threshold value iscalculated by the estimation threshold functionas shown in Equation (14). In their paper, Zhang \& Gunturk [24] identifiesthe linear relation between the characteristic of intensity domain $\sigma_{\mathrm{r}}$ and the noise standard deviation $\sigma_{\mathrm{n}}$. The optimal value of the parameter $\sigma_{r}$ is related to $\sigma_{n}$ with scale factor sas expressed by the following equation:

$$
\sigma_{\mathrm{r}}=\mathrm{s}^{*} \sigma_{\mathrm{n}}
$$

Meanwhile, $\sigma_{n}$ is defined as a ratio between the median absolute deviation (MAD) of the detail subband (HH) coefficients and a decimal number 0.6745 .

\subsection{Data}

In this research, we used two kinds of imagesfor each experiment. The first type was a phantom image with the size of $200 \times 200$, the modified Shepp-Logan, that was generated by the phantom function in Matlab 2013 as shown in Figure 2(a). This phantom image was added with speckle noise for noise variance equal to 0.1, as shown in Figure 2(b). The other image was the real abdomen ultrasound image that was obtained using a portable scanner, i.e. the SonoStar UBox-10 Ultrasound B Scanner with a transabdominal convex probe $3.5 \mathrm{MHz}$. The example of the real fetal abdomen image is presented in Figure 3.

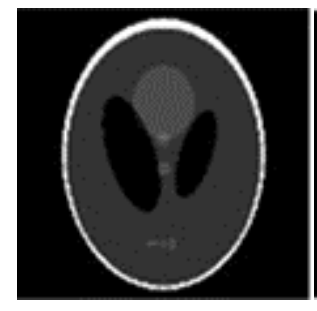

(a)

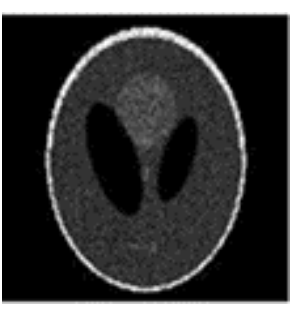

(b)
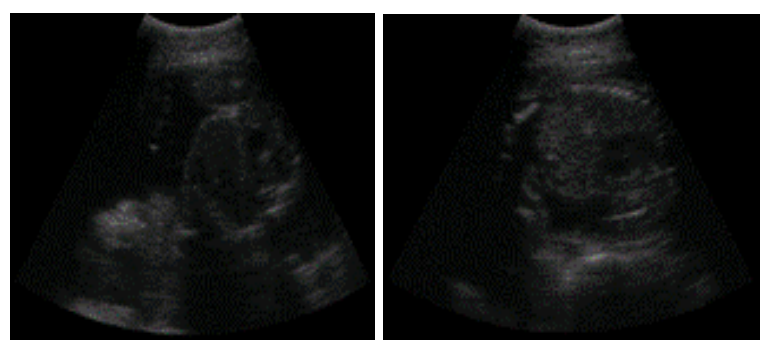

Figure 2. (a) Phantomimage b) Noisy phantom

Figure 3. Example of the fetal abdomen ultrasound image image

\subsection{Proposed speckle noise reduction method}

The proposed method for ultrasound image denoising described in Figure 4 includes five processes: the discrete wavelet transform (DWT), bilateral filtering, the thresholding process followed by anisotropic diffusion, and the inverse wavelet transform (IDWT). Firstly, the noisy image is decomposed into the $n$th 
level by the discrete wavelet transform (DWT) that generates a subband which is an approximation of the original image and the three sub-bands that provides detailed information on the vertical, horizontal and diagonal of the original image. Mother wavelet function applied in this method is a Daubechies ' $\mathrm{db} 8$ ' function [29]. The approximation subband is processed by bilateral filtering in the Equation (5). The detail subbands are computed using the soft thresholding technique as in Equation (13) and followed by the anisotropic diffusion method with the second diffusion function as in Equation (11). To estimate thethreshold value, we use the formula in Equation (14). Unlike other existing anisotropic diffusion in [19] and [20], we apply the derivative filters for eight directions at the derivative approximationstage of the anisotropic diffusion process. The anisotropic diffusion process can increase noise removal in detail subbands. After all the process, the inverse wavelet transform is applied to get an approximation image. All of these steps arerepeated using the approximation image as the input until it reaches the first level decomposition wavelet. Figure 4 shows an example of denoising image for level $n=1$. To achieve the best result, we determine the parameters required in this method as shown in Table 1.

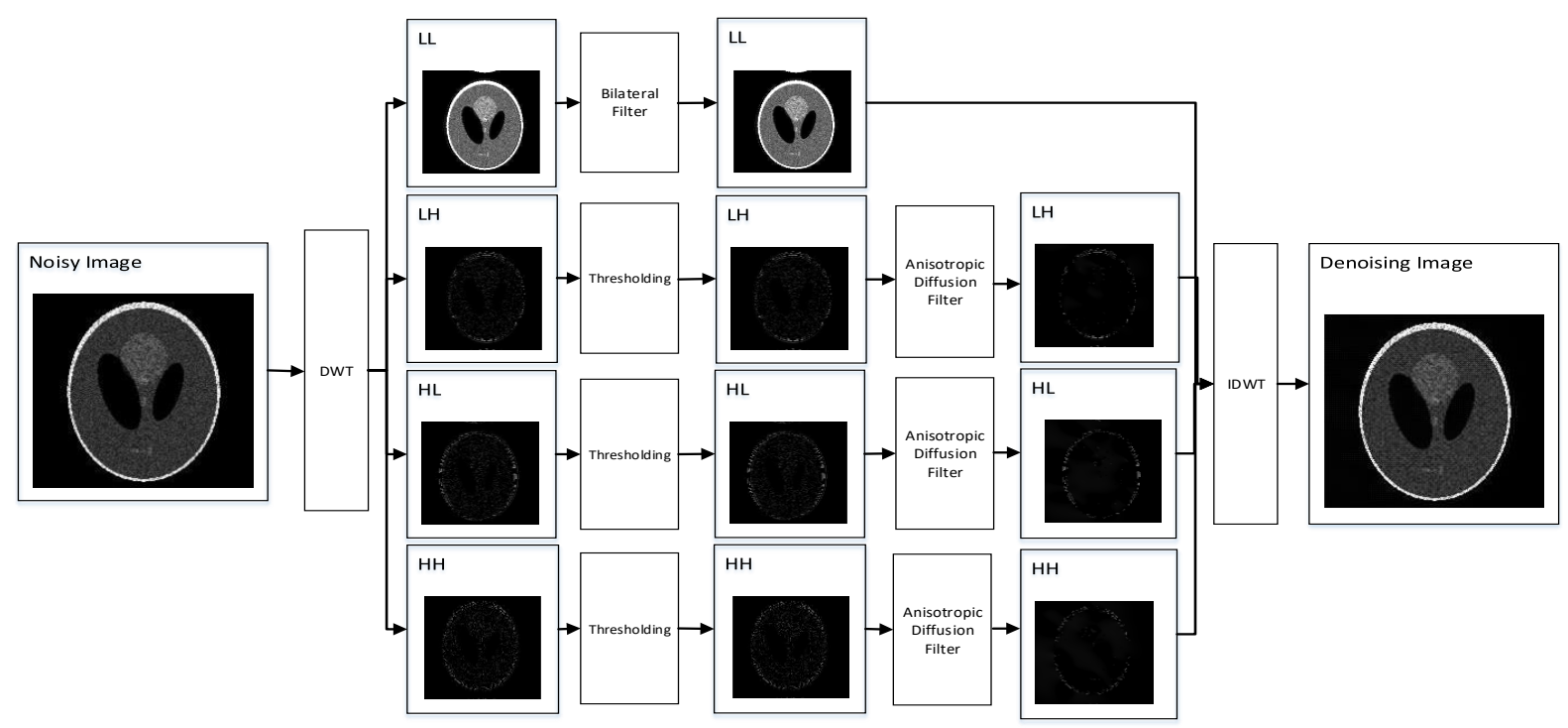

Figure 4. The framework of the proposed speckle noise reductionmethod for level $n=1$

Table 1. The ParameterSetting of the Proposed Method

\begin{tabular}{lc}
\multicolumn{1}{c}{ Parameter } & Value \\
\hline Window size & $5 \times 5$ \\
Standard deviation $\sigma_{d}$ & 3 \\
Noise standard deviation $\sigma_{n}$ & 3 \\
Standard deviation $\sigma_{r}$ & $7 * \sigma_{n}$ \\
Step size $\Delta t$ & 0,1 \\
Iteration $n$ & 50 \\
Gradient threshold $k$ & 5 \\
Diffusion equation & Equation $(11)$ \\
Wavelet type & $\mathrm{db} 8$ \\
Decomposition level & 3 \\
\hline
\end{tabular}

\subsection{Fetal abdominal circumference segmentation method}

The fetal abdomen segmentation in an ultrasound image is used to get the abdominal circumference (AC) with standard measurement as presented in Figure 5 where the left figure shows the landmark of the abdomen image in the right figure. The landmark contains (1) umbilical vein (2) middle portal vein (3) stomach (4) spine (5) rib. The abdominal circumference is measured around the outside of the skin line as shown by white circle in Figure 5 [30].

We apply the active geometric shape model (AGSM) method that is proposed by Wang \& Boyer [12] to detect and to measure the fetal abdominal circumference. The AGSM method implements a geometric shape approach represented by parametric equations to fit an object in an image. This technique adjusts the 
shape parameters according to the integral of a force field along the shape contour. The output of the AGSM function is the radius of a generated circle that applied to calculate the circumference. Figure 6 shows the block diagram of the abdominal circumference segmentation process.

The input images are the ultrasound image from denoising process that cropped automatically. At the first step, the Canny edge detector is applied to get the boundaries in the binary image and afterwards the result is processed by the Gaussian smoothing filter. Furthermore, the gradient vector field (GVF) isderivedfrom the blurred image in the vertical direction and horizontal direction. Based on the two vector fields and initial circle parameters, a circle equation is updated.

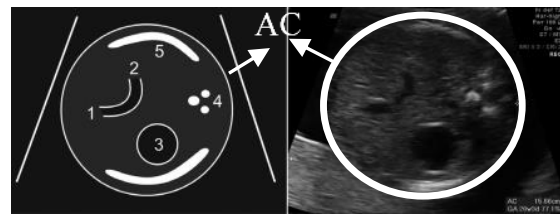

Figure 5.Standard of AC measurement on 2D ultrasound images [30]

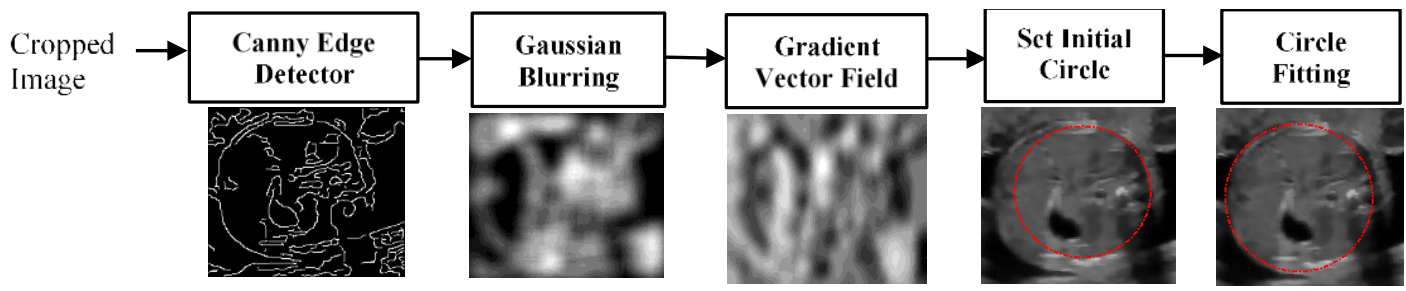

Figure 6. The block diagram of abdominal circumference segmentation

\subsection{Quantitative quality measurements}

In this research, we used four quality performance in the first experiment, i.e., the peak signal to noise ratio (PSNR) [3], the root mean square error (RMSE) [3], the Pratt's Figure Of Merit (FOM) and the Mean Structural Similarity Index Measure (MSSIM). The MSSIM that proposed by [31] is a measurement used to measure the image restoration results quality that corresponds to the human visual perception by combining three common factors that are the loss of correlation, luminance and contrast distortion. The Pratt's figure of merit (FOM) that submitted by [20] is a measurement to compare edge preservation performance of different speckle reduction schemes. Results of FOM depend on the edge detection method used. In this research, we implemented the Canny Edge Detector.

In the segmentation experiment, we measured the performance using the accuracyand the Dice similarityas used in [13]. Suppose $O_{S R}^{M}$ denotes the result of segmentation for method $M$ and $O_{G T}$ is ground truth obtained from manual segmentation. The accuracyis the average of two values, i.e., True Positive (TP) and True Negative (TN). TP is formulated as the ratio of the intersection between $O_{S R}^{M}$ and $O_{G T}$ to the region covered by $O_{G T}$. TN is the ratio of the region not covered by $O_{S R}^{M}$ and $O_{G T}$ to the areas outside the region $O_{G T}$. True-Positive Ratio (TPR) also known as sensitivity and True-Negative Ratio (TNR) also known as specificity [6]. Dice similarity (D) is the primary similarity metric used to measure overlapping between two regions spatially, defined as follows.

$$
D=\frac{2\left|O_{S R}^{M} \cap O_{G T}\right|}{\left|O_{G T}\right|+\left|O_{S R}^{M}\right|}
$$

\section{RESULTS AND ANALYSIS}

This section presents the denoising resultevaluation to analyze the proposed method validation. To evaluate this method, we compare it to the existing method. The parameter setting of the existing methods is presented in Table 2. 
Table 2. The Parameter Setting of the Compared Denoising Methods

\begin{tabular}{lll}
\hline \multicolumn{1}{c}{ Method } & \multicolumn{1}{c}{ Category } & \multicolumn{1}{c}{ Parameter Setting } \\
\hline Lee Filter [16] & Linear filter & Window size 7x7 \\
Frost Filter [17] & Linear filter & Window size 7x7 \\
Shock Filter [3] & Non-linear filter & Window size 9x9; step size dt $=0,25$; iteration $n=30$ \\
Bilateral Filter [18] & Non-linear filter & Windowsize 5x5; standard deviation $\sigma_{d}=3 ;$ noise standard deviation $\sigma_{n}=$ \\
& & $3 ;$ standard deviation $\sigma_{r}=7 * \sigma_{n}$ \\
AD [19] & Diffusion filter & $\begin{array}{l}\text { Step size } \Delta t=0,1 ; \text { iteration } n=50 ; \text { gradient threshold } k=5 \text {; diffusion } \\
\text { equation in Equation }(11)\end{array}$ \\
SRAD [15] & Diffusion filter & Step size $\Delta t=0,25 ;$ iteration $n=40$ \\
DWT [27] & Multiresolution & Decomposition level=3; wavelet type $=$ 'db8' \\
MBF [24] & Hybrid & Decomposition level=3; wavelet type $=$ 'db8'; window size 5x5; standard \\
& & deviation $\sigma_{d}=3 ;$ noise standard deviation $\sigma_{n}=3$; standard deviation \\
& & $\sigma_{r}=7 * \sigma_{n}$ \\
\hline
\end{tabular}

\subsection{Reduction noise performance}

In the first experiment, we compared our method with existing methods using the parameters that contained in Table 2 with the PSNR, RMSE, MSSIM, and Pratt's FOM quantitative measurements. To obtainthe Pratt's FOM, we applied the Canny edge detector with the standard deviation of the Gaussian filter $\sigma=\sqrt{2}$ to the original phantom image and noisy phantom image.

Table 3 summarizes the comparis on of the quality measurement results from various methods. The bilateral filtering method has the best value of the mean square error and the peak signal to ratio values. Unfortunately, from the Pratt's FOMvalue, the bilateral filterability to maintain the edge is lower than other methods. Nevertheless, usage of bilateral filtering will be able to maintain the visual structure that corresponds to human visual perception, as seen from the MSSIM. The thresholding approach applied to the multiresolution wavelet based method can keep the average error small in the image restoration process, as seen from the RMSE value. Therefore, the integration of the bilateral filter, the anisotropic diffusion and the thresholding wavelet in our proposed method offer the best performance quantitatively. The MSSIM index value of the proposed method gives a significant improvement. Consequently, the proposed method result has a great similarity structurallyto the reference image.Based on the Pratt's FOM, this method provides a better value. Therefore, it indicates that the method is able to preserve the edge. Frost filter result has the high Pratt's FOM value, conversely, the PSNR and the RMSE value of the Frost filter methodare worse than the proposed method. Visually in Figure 7, the proposed method gives the best image that can reduce noise and remain preserved the edges. In Figure 7(c), Frost filter result looks more blurred, such that the features or the edges of the image are lost.

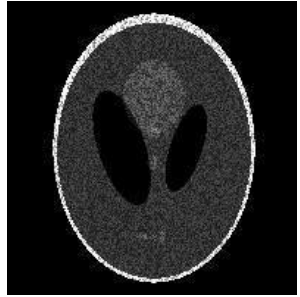

(a)

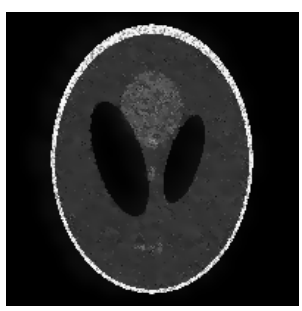

(f)

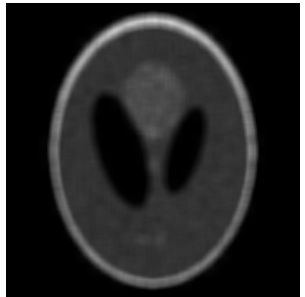

(b)

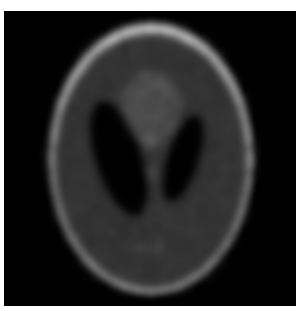

(g)

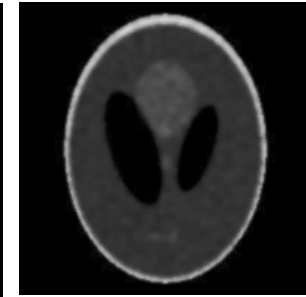

(c)

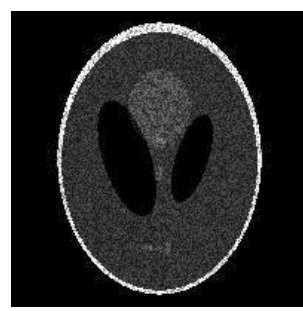

(h)

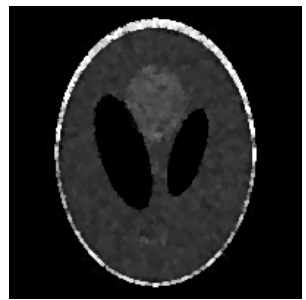

(d)

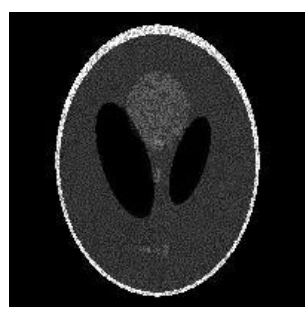

(i)

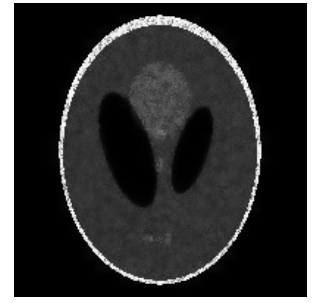

(e)

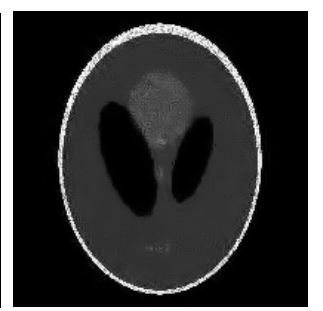

(j)

Figure 7. The denoisingresultof (a) noisy image using (b) Lee filter, (c) Frost filter, (d) shock filter, (e) bilateral filter, (f) anis otropic diffusion, (g) SRAD,(h) discrete wavelet transform,

(i) multiresolution bilateral filtering and (j) the proposed method 
Table 3. The Performance of Different Speckle Reduction Techniques

\begin{tabular}{lcccc}
\hline \multicolumn{1}{c}{ Method } & RMSE & PSNR & MSSIM & Pratt's FOM \\
\hline Lee Filter [16] & 30,429 & 21,475 & 0,783 & 86,125 \\
Frost Filter [17] & 22,597 & 24,060 & 0,880 & 92,607 \\
Shock Filter [3] & 16,582 & 26,748 & 0,877 & 57,899 \\
Bilateral Filter [18] & 11,776 & 29,721 & 0,882 & 52,816 \\
AD [19] & 12,656 & 29,095 & 0,780 & 64,688 \\
SRAD [15] & 32,246 & 20,972 & 0,757 & 82,122 \\
DWT [27] & 16,274 & 26,911 & 0,701 & 36,952 \\
MBF [24] & 16,405 & 26,842 & 0,861 & 78,267 \\
Proposed Method & 12,561 & 29,160 & 0,903 & 93,027 \\
\hline
\end{tabular}

\subsection{Abdominal circumference segmentation performance}

Thesecond experiment aimed to investigate the reduction noise effect for detecting abdominal circumference in a fetal ultrasound image. The example result of abdominal circumference segmentation in Figure 8 shows that the proposed noise reduction method provides a significant improvement compared to the other methods. The ultrasound image used in Figure 8 obtained from portable scanners with low image quality.The proposed method at Figure 8(j) provides the best result with the accuracy of 0,9237 . It is followed by the multiresolution bilateral filtering (Figure 8(i)) with the accuracy of 0,9031 . The accuracy of the anisotropic diffusion method in Figure 8(f) and the SRAD in Figure 8(g) arenot relatively different that are 0,8852 and 0,8841 . While the accuracy of the linear filter, the Lee filter (Figure $8(\mathrm{~b})$ ) and the Frost filter (Figure $8(\mathrm{c})$ ) are equal to 0,8777 and 0,8785 . For the nonlinear filter method, the accuracy of the shock filter ((Figure $8(\mathrm{~d}))$ is 0,8816 and the bilateral filter ((Figure $8(\mathrm{e}))$ is 0,8774 .

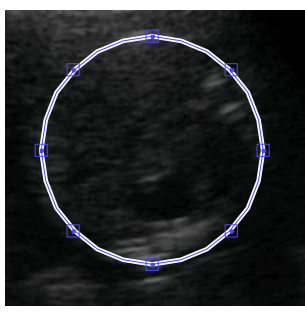

(a)

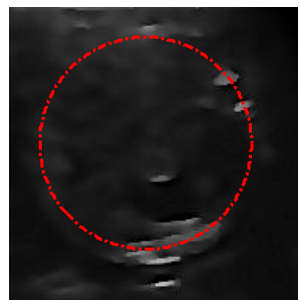

(f)

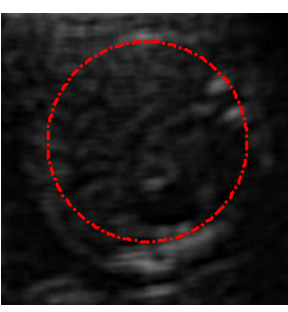

(b)

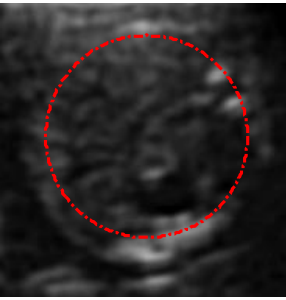

(g)

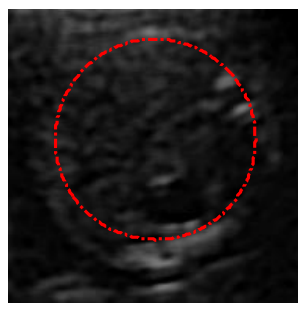

(c)

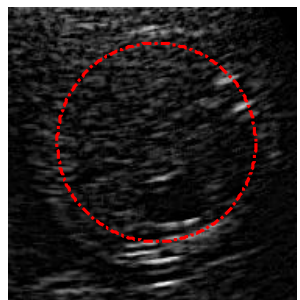

(h)

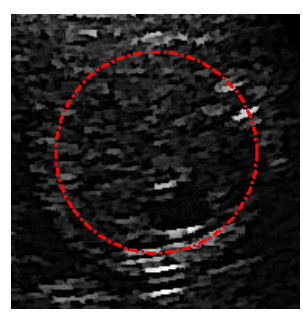

(d)

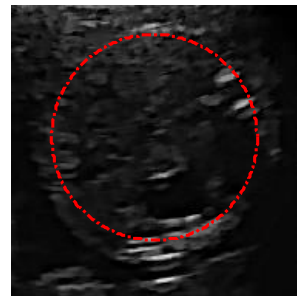

(i)

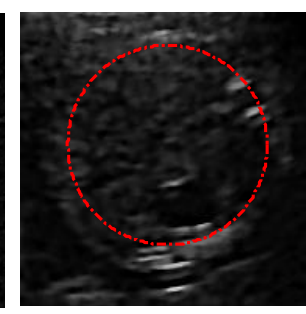

(e)

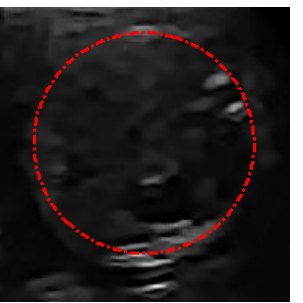

(j)

Figure 8. The segmentationresults of the fetal abdominal image using (a) manual segmentation, (b) the Lee filter, (c) Frost filter, (d) shock filter, (e) bilateral filter, (f) anisotropic diffusion, (g) SRAD, (h) discrete wavelet transform, (i)multiresolution bilateral filtering and (j) the proposed method

The mean and the standard deviation of the performance in term of accuracy, sensitivity, specificity and Dice similarity are presented in Figure 9. In terms of accuracy, sensitivity and Dice similarity, the proposed method performed better than the other methods. The standard deviation of the proposed method appears quite small. This might be due to the ability of the proposed method to improve the various types and quality of ultrasound images. In terms of specificity, the discrete wavelet transform and the bilateral filtering have the best value but close to the others method. Table 4 summarizes the overall performance of the abdominal circumference segmentation task. The proposed method has the best accuracy, with that mean is equal to 0,9237 and the standard deviation is equal to 0,037 . Meanwhile, all methods have almost the same 
specificity value. From the comparative results, it can be concluded that the proposed noise reduction method can improve the accuracy of fetal biometric detection and measurement.

Table 4. The Average Performance of Abdominal Circumference Segmentation using Different Speckle Reduction Techniques

\begin{tabular}{lcccc}
\hline \multicolumn{1}{c}{ Method } & Accuracy & Sensitivity & Specificity & Dice Similarity \\
\hline Lee Filter [16] & $0,855 \pm 0,099$ & $0,723 \pm 0,209$ & $0,986 \pm 0,019$ & $0,817 \pm 0,154$ \\
Frost Filter [17] & $0,864 \pm 0,089$ & $0,738 \pm 0,185$ & $0,989 \pm 0,015$ & $0,832 \pm 0,133$ \\
Shock Filter [3] & $0,829 \pm 0,086$ & $0,667 \pm 0,179$ & $0,991 \pm 0,014$ & $0,782 \pm 0,129$ \\
Bilateral Filter [18] & $0,838 \pm 0,103$ & $0,684 \pm 0,215$ & $0,992 \pm 0,014$ & $0,790 \pm 0,167$ \\
AD [19] & $0,862 \pm 0,074$ & $0,751 \pm 0,157$ & $0,973 \pm 0,015$ & $0,837 \pm 0,108$ \\
SRAD [15] & $0,887 \pm 0,072$ & $0,786 \pm 0,150$ & $0,987 \pm 0,018$ & $0,864 \pm 0,101$ \\
DWT [27] & $0,827 \pm 0,103$ & $0,663 \pm 0,211$ & $0,992 \pm 0,013$ & $0,772 \pm 0,167$ \\
MBF [24] & $0,902 \pm 0,063$ & $0,820 \pm 0,122$ & $0,983 \pm 0,019$ & $0,888 \pm 0,079$ \\
Proposed Method & $0,929 \pm 0,037$ & $0,872 \pm 0,075$ & $0,986 \pm 0,013$ & $0,923 \pm 0,037$ \\
\hline
\end{tabular}

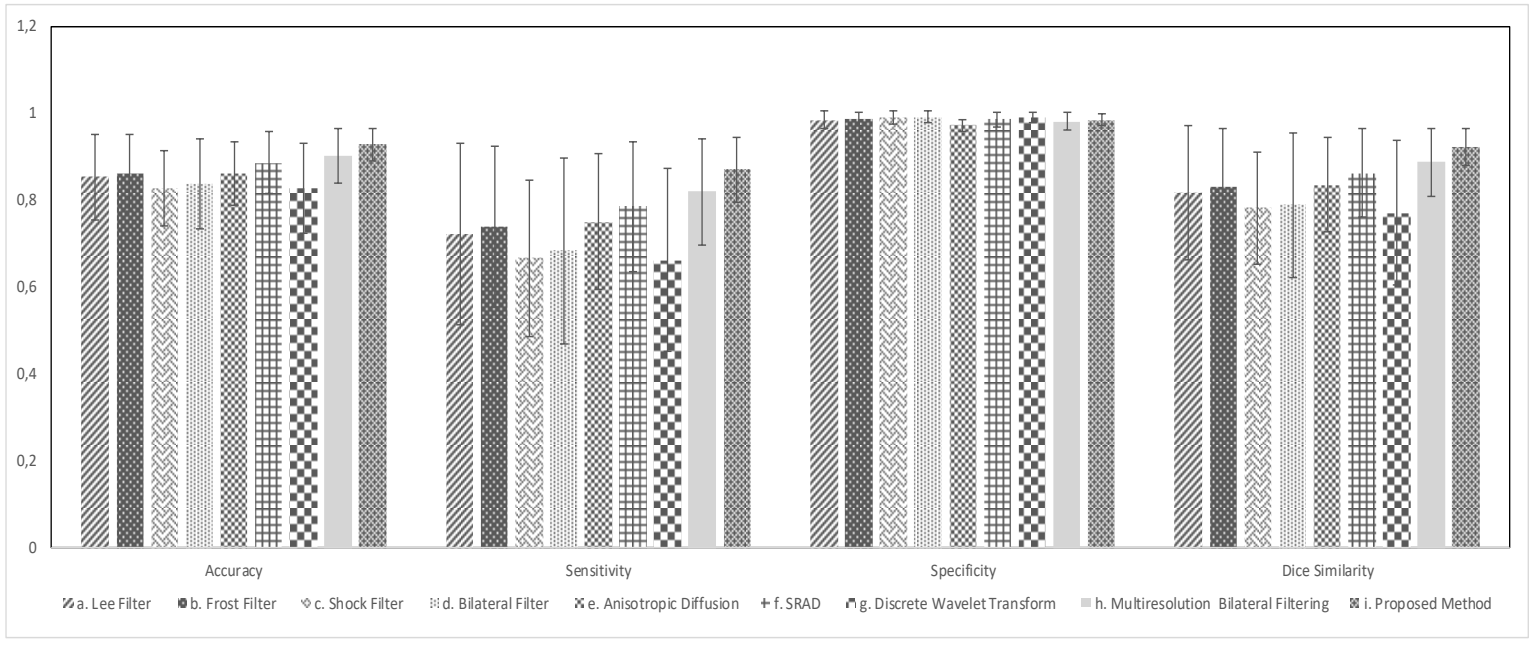

Figure 9. The mean and standart deviation of the abdominal circumference segmentation for each noise reduction methods in term accuracy, sensitivity, specificity and dice similarity

\section{CONCLUSION}

The proposed denoising method was a hybrid speckle noise reduction framework that combinedthe anisotropic diffusion method and multiresolution bilateral filtering method. In this proposed scheme, the wavelet thresholding technique and anisotropic diffusion method were applied to the detail subbands of the wavelet decomposition. Meanwhile, the approximation subband was processed by the bilateral filtering method. To find the performance of the proposed approach, we compared it with the other denoising techniques, such as the Lee filter, Frost filter, shock filter, bilateral filter, wavelet thresholding, and multiresolution bilateral filtering method. The comparison was made regarding noise reduction and the effect of noise reduction results to improve the abdominal circumference segmentation performance in fetal ultrasound images. From Pratt's FOM measurement, this method obtained the best performance to remove speckle noise without reducing the edge. Moreover, this approach also had the best quality of human visual perception. For the segmentation task, the proposed denoising method could improve the accuracy of the fetal biometric abdominal circumference segmentation. The segmentation performance variance of the intended image is quite small, so it means that the proposed method can enhance the various types and quality of ultrasound images. For further development, an attempt to integrate other wavelet functions, such as the complex wavelet transform, rational dilation wavelet transform, or stationary wavelet transform.

\section{REFERENCES}

[1] Contreras Ortiz SH, Chiu T, Fox MD, "Ultrasound image enhancement: A review”, Biomedical Signal Processing and Control. 2012, vol. 7, no. 5, pp. 419-428.

[2] Hermawati FA, Sugiono, Evonda, "Ultrasound Image Formation from Doppler Transducer", In: Chang SH, Parinov 
IA, Jani MA (eds), Advanced Materials Techniques, Physics, Mechanics and Applications, Springer International Publishing, 2017, pp. 535-543.

[3] Sahu S, Dubey M, Khan MI, "Comparative Analysis of Image Enhancement Techniques for Ultrasound Liver Image", International Journal of Electrical and Computer Engineering, 2012, vol. 2, no. 6, pp. $792-797$.

[4] Chen Y, Zhou XM, Liu DC, "Localizing Region-Based Level-set Contouring for Common Carotid Artery in Ultrasonography", Telecommunication Computing Electronics and Control, 2013, vol. 11, no. 4, pp. 791-796.

[5] Sushma SJ, Prasanna Kumar SC, "Advancement in Research Techniques on Medical Imaging Processing for Breast Cancer Detection", International Journal of Electrical and Computer Engineering, 2016, vol. 6, no. 2, pp. 717.

[6] Lestari DP, Madenda S, Wibowo EP, "Comparison of Three Segmentation Methods for Breast Ultrasound Images Based on Level Set and Morphological Operations", International Journal of Electrical and Computer Engineering, 2017, vol. 7, no. 1, pp. 383-391.

[7] Lee HJ, Song DH, Kim KB, "Effective Computer-Assisted Automatic Cervical Vertebrae Extraction with Rehabilitative Ultrasound Imaging by using K-means Clustering", International Journal of Electrical and Computer Engineering, 2016, vol. 6, no. 6, pp. 2810-2817.

[8] Espinoza J, Good S, Russell E, Lee W, "Does the Use of Automated Fetal Biometry Improve Clinical", Journal of Ultrasound in Medicine, 2013, vol. 32, no. 5, pp. 847-850.

[9] Yu J, Wang Y, Chen P, Shen Y, "Fetal Abdominal Contour Extraction and Measurement in Ultrasound Images", Ultrasound in Medicine and Biology, 2008, vol. 34, no. 2, pp. 169-182.

[10] Wang W, Qin J, Zhu L, Ni D, Chui YP, Heng PA, "Detection and measurement of fetal abdominal contour in ultrasound images via local phase information and iterative randomized hough transform", Bio-Medical Materials and Engineering, 2014, vol. 24, no. 1, pp. 1261-1267.

[11] Kraitem Z, Saii M, "Automatic Detection and Measurement of Abdominal Circumference in Fetal Ultrasound Images", International Journal of Information Research and Review, 2016, vol. 3, no. 3, pp. 1997-2000.

[12] Wang Q, Boyer KL, "The Active Geometric Shape Model: A New Robust Deformable Shape Model and Its Applications", Computer Vision and Image Understanding, 2012, vol. 116, no. 12, pp. 1178-1194.

[13] Rueda S, Fathima S, Knight CL, Yaqub M, Papageorghiou AT, Rahmatullah B, et al, "Evaluation and Comparison of Current Fetal Ultrasound Image Segmentation Methods for Biometric Measurements: A Grand Challenge", IEEE Transactions On Medical Imaging, 2014, vol. 33, no. 4, pp. 797-813.

[14] Joel T, Sivakumar R, "Despeckling of Ultrasound Medical Images: A Survey”, Journal of Image and Graphics, 2013, vol. 1, no. 3, pp. 161-165.

[15] Loizou CP, Pattichis CS, Despeckle Filtering for Ultrasound Imaging and Video, Volume I: Algorithms and Software. Second Ed. Morgan \& Clay pool Publishers; 2015.

[16] Lee J-S, "Digital Image Enhancement and Noise Filtering by Use of Local Statistics", IEEE Transactions Pattern Analysis and Machine Intelligence, 1980, vol. PAMI-2, no. 2, pp. 165-168.

[17] Frost VS, Stiles JA, Shanmugan KS, Holtzman JC, "A Model for Radar Images and Its Application to Adaptive Digital Filtering of Multiplicative Noise", IEEE Transaction Pattern Analysis and Machine Intelligence, 1982, vol. PAMI-4, no. 2, pp. 157-166.

[18] Paris S, Kornprobst P, Tumblin J, Durand F, Paris S, Kornprobst P, et al., "Bilateral Filtering: Theory and Applications", Computer Graphics and Vision, 2008, vol. 4, no. 1, pp. 1-73.

[19] Perona P, Malik J. Scale-Space and Edge Detection Using Anisotropic Diffusion. IEEE Transactions on Pattern Analysis and Machine Intelligence. 1990; 12 (7): 629-639.

[20] Yu Y, Acton ST, "Speckle Reducing Anisotropic Diffusion", IEEE Transactions on Image Processing, 2002, vol. 11, no. 11, pp. 1260-1270.

[21] Babu Y MM, Subramanyam M, Giri Prasad M, “A New Approach for SAR Image Denoising”, International Journal of Electrical and Computer Engineering, 2015, vol. 5, no. 5, pp. 984-991.

[22] Gopinathan S, Kokila R, Thangavel P, "Wavelet and FFT Based Image Denoising Using Non-Linear Filters", International Journal of Electrical and Computer Engineering, 2015, vol. 5, no. 5.

[23] Hamiane M, Saeed F, "SVM Classification of MRI Brain Images for Computer- Assisted Diagnosis", International Journal of Electrical and Computer Engineering, 2017, vol. 7, no. 5, pp. 2555-2564.

[24] Zhang M, Gunturk BK, "Multiresolution Bilateral Filtering for Image Denoising", IEEE Transactions on Image Processing, 2008, vol. 17, no. 12, pp. 2324-2333.

[25] Zhang J, Lin G, Wu L, Wang C, Cheng Y, "Wavelet and fast bilateral filter based de-speckling method for medical ultrasound images", Biomedical Signal Processing and Control, 2015, vol. 18, no. 1, pp. 1-10.

[26] Weickert J, “Coherence-Enhancing Shock Filters”, In: DAGM-Symposium 2003, 2003, pp. 1-8.

[27] Şendur L, Selesnick IW, "Bivariate Shrinkage Functions for Wavelet-Based Denoising Exploiting Interscale Dependency", IEEE Transactions on Signal Processing, 2002, vol. 50, no. 11, pp. 2744-2756.

[28] Donoho DL, "De-Noising by Soft-Thresholding", IEEE Transactions on Information Theory, 1995, vol. 41, no. 3, pp. 613-627.

[29] Adamo F, Andria G, Attivissimo F, Maria A, Lanzolla L, Spadavecchia M, "A Comparative Study on Mother Wavelet Selection in Ultrasound Image Denoising", Measurement, 2013, vol. 46, no. 8, pp. 2447-2456.

[30] Bethune M, Alibrahim E, Davies B, Yong E, "A Pictorial Guide for the Second Trimester Ultrasound", Australasian Journal of Ultrasound in Medicine, 2013, vol. 16, no. 3, pp. 98-113.

[31] Wang Z, Bovik AC, Sheikh HR, Member S, Simoncelli EP, Member S, "Image Quality Assessment: From Error Visibility to Structural Similarity”, IEEE Transactions on Image Processing, 2004, vol. 13, no. 4, pp. 600-612. 